\title{
Fault Diagnosis of Rotating Machinery Based on Adaptive Stochastic Resonance and AMD-EEMD
}

\author{
Peiming Shi, ${ }^{1}$ Cuijiao Su, ${ }^{1}$ and Dongying Han ${ }^{2}$ \\ ${ }^{1}$ Institute of Electrical Engineering, Yanshan University, Qinhuangdao, Hebei 066004, China \\ ${ }^{2}$ Institute of Vehicles and Energy, Yanshan University, Qinhuangdao, Hebei 066004, China \\ Correspondence should be addressed to Peiming Shi; peiming.shi@163.com
}

Received 4 November 2015; Revised 15 December 2015; Accepted 5 January 2016

Academic Editor: Juan P. Amezquita-Sanchez

Copyright (c) 2016 Peiming Shi et al. This is an open access article distributed under the Creative Commons Attribution License, which permits unrestricted use, distribution, and reproduction in any medium, provided the original work is properly cited.

\begin{abstract}
An adaptive stochastic resonance and analytical mode decomposition-ensemble empirical mode decomposition (AMD-EEMD) method is proposed for fault diagnosis of rotating machinery in this paper. Firstly, the stochastic resonance system is optimized by particle swarm optimization (PSO), and the best structure parameters are obtained. Then, the signal with noise is put into the stochastic resonance system and denoising and enhancing the signal. Secondly, the signal output from the stochastic resonance system is extracted by analytical mode decomposition (AMD) method. Finally, the signal is decomposed by ensemble empirical mode decomposition (EEMD) method. The simulation results show that the optimal stochastic resonance system can effectively improve the signal-to-noise ratio, and the number of effective components of EEMD decomposition is significantly reduced after using AMD, thus improving the decomposition results of EEMD and enhancing the amplitude of components frequency. Through the extraction of the rolling bearing fault signal feature proved that the method has a good effect.
\end{abstract}

\section{Introduction}

Rotating machinery plays a significant role in a wide range of industrial applications, such as aerospace, transportation vehicles, and power generators. The rotating machine faults can cause violent vibration on the machine and even endanger normal machine operation. Therefore, accurate health monitoring and diagnosis system is needed to identify incipient fault that may occur in a rotating machine [14]. However, the defect-induced fault signal of the rotating machine is often corrupted by the noise coming from other coupled machine components and working environment, which makes some incipient faults not easy to be recognized. The challenge of fault recognition requires enhancing the weak fault information from heavy background noise.

Stochastic resonance (SR) theory was proposed by Benzi et al. in 1981 to explain the periodicity of the Earth's ice ages [5]. It can make part of the noise energy transfer to the low frequency signal through a nonlinear system, so weak signal drowned in the noise has been greatly strengthened at the same time to reduce the noise, and greatly improve the output signal-to-noise ratio (SNR) [6-11]. Therefore the stochastic resonance method has strong immunity to noise and can effectively extract weak signal under strong noise background. Stochastic resonance is actually the optimal matching relationship between signal, noise, and nonlinear system. However, the amplitude of the signal and the noise intensity may change over time in the actual engineering, so it is necessary to automatically adjust the nonlinear system parameters according to the different signal, in order to achieve stochastic resonance and improve the output SNR.

Ensemble empirical mode decomposition (EEMD) is a noise-assisted data analysis method which was proposed based on empirical mode decomposition (EMD) [12] by Huang et al. This method uses the Gauss white noise that has the statistical characteristics of frequency distribution; the signal after adding the Gauss white noise is continuous in different scales. It can effectively solve the mode mixing problem of EMD and successfully applied in some engineering practice [13-17].

Analytical modal decomposition (AMD) method was proposed by Chen and Wang [18]. This method can separate 
different frequency components from the signal, but the premise is to know the frequency components in the signal and then determine the bisecting frequency. Therefore, if one wants to extract the known part of the signal, the signal can be processed by AMD method.

A method of rotating machinery fault diagnosis based on adaptive stochastic resonance and AMD-EEMD is proposed in this paper. Firstly, the bistable stochastic resonance system is optimized by particle swarm optimization (PSO) and putting the signal which containing noise into the stochastic resonance system and then denoising and enhancing the signal. Secondly, the signal output from the stochastic resonance system is extracted by AMD. Finally, the signal is decomposed by EEMD method. The simulation results show that the optimal stochastic resonance system can effectively improve the SNR, and the number of effective components of EEMD decomposition is significantly reduced after using AMD, thus improving the decomposition results of EEMD and enhancing the amplitude of components frequency.

The rest of the paper is organized as follows. The classical bistable SR theory, the PSO algorithm, and the theories of EEMD and AMD are given and the SR-AMD-EEMD method is proposed in Section 2. Section 3 describes the advantage of the proposed method by simulation experiment. The adaptive stochastic resonance and AMD-EEMD method is applied to the fault diagnosis of a rolling bearing in Section 4, and the application result proves the effectiveness of the proposed method. Finally, Section 5 provides the conclusion.

\section{Adaptive Stochastic Resonance and AMD-EEMD Algorithm}

2.1. Bistable Stochastic Resonance Model. Stochastic resonance is synergistic effect of the input signal and the noise signal on the nonlinear system with certain parameters, and then part of the energy of noise signal transfers to the input signal, which can improve the SNR to achieve the purpose of weak signal recognition.

The Langevin equation is to describe the typical model of nonlinear bistable system [19-21]. The Langevin equation of bistable system is considered as follows:

$$
\begin{aligned}
\frac{d x}{d t} & =-U^{\prime}(x)+S(t)+N(t) \\
& =a x-b x^{3}+S(t)+N(t),
\end{aligned}
$$

where $a$ and $b$ are the structural parameters of the bistable system, $S(t)$ is the input signal, and $N(t)$ is the noise signal. Let $\langle N(t)\rangle=0,\langle N(t) N(t-\tau)\rangle=2 D \delta(t)$, and $N(t)=$ $\sqrt{2 D} \xi(t)$, where $\xi(t)$ is white noise with zero mean and unit variance and $D$ is the noise intensity. The potential function for the above bistable system can be denoted as

$$
U(x)=-\frac{1}{2} a x^{2}+\frac{1}{4} b x^{4} .
$$

The above equation has two minimum values in $x=$ $\pm \sqrt{a / b}$ and has a maximum value in $x=0$, corresponding to the system of two lowest points and a barrier point, corresponding to the two potential hydrazine points and a barrier point of the system. And its barrier height is $\Delta U=$ $a^{2} /(4 b)$.

2.2. Particle Swarm Optimization Algorithm. Particle swarm optimization (PSO) algorithm is an evolutionary computing technology proposed by Eberhart and Kennedy. Assuming $i$ particle position and velocity in $d$ dimensional search space are $X^{i}=\left(x_{i 1}, x_{i 2}, \ldots, x_{i d}\right)$ and $V^{i}=\left(v_{i 1}, v_{i 2}, \ldots, v_{i d}\right)$, respectively, in each iteration, particles update their own by tracking two optimal solutions, one is the optimal solution $P^{i}=\left(p_{i 1}, p_{i 2}, \ldots, p_{i d}\right)$ found by particle itself, and the other is the optimal solution of current population $P_{g}$. When finding the two best values, the particle updates its velocity and position according to the following formula:

$$
\begin{aligned}
v_{i, j}(t+1)= & \omega v_{i, j}(t)+c_{1} r_{1}\left\lfloor P_{i, j}-x_{i, j}(t)\right\rfloor \\
& +c_{2} r_{2}\left\lfloor P_{g, j}-x_{i, j}(t)\right\rfloor, \\
x_{i, j}(t+1)= & x_{i, j}(t)+v_{i, j}(t+1), \quad j=1,2, \ldots, d,
\end{aligned}
$$

where $\omega$ is inertia weight, $c_{1}$ and $c_{2}$ are the learning factors, and $r_{1}$ and $r_{2}$ are random numbers between 0 and 1 uniform distribution $[22,23]$.

Because the larger weights are conducive to jumping out of the local minimum value, they are suitable for global search. And smaller weights are conducive for accurate local search in the current search area, which is helpful in the convergence of the algorithm.

Therefore, in order to prevent the phenomenon of premature convergence and oscillation occurred in the vicinity of the global optimal solution of PSO algorithm, you can use the linear decreasing weight as follows:

$$
\omega=\omega_{\max }-\frac{t *\left(\omega_{\max }-\omega_{\min }\right)}{t_{\max }},
$$

where $\omega_{\max }$ and $\omega_{\min }$ are maximum value and minimum value of $\omega, t$ is the current number of iterations, and $t_{\max }$ is the maximum number of iterations. Usually, $\omega_{\max }=0.9$ and $\omega_{\min }=0.4$.

2.3. EEMD Based on AMD. A new signal decomposition method called AMD was proposed by Chen and Wang in 2012 [18]. This method can decompose signals with closely spaced frequency components, which means being able to extract the signal.

If a time series $x(t)$ is divided into two signals by bisecting frequency $\omega_{b}$

$$
x(t)=s_{1}(t)+\bar{s}_{1}(t) .
$$

We can get that

$$
\begin{aligned}
s_{1}(t)= & \sin \left(\omega_{b} t\right) H\left[x(t) \cos \left(\omega_{b} t\right)\right] \\
& -\cos \left(\omega_{b} t\right) H\left[x(t) \sin \left(\omega_{b} t\right)\right], \\
\bar{s}_{1}(t)= & x(t)-s_{1}(t),
\end{aligned}
$$


where $H[\cdot]$ is Hilbert transformation of the function in brackets.

So the original signal will get the two signals by using the AMD method.

The essence of EEMD method is repeated EMD decomposition added Gauss white noise. Finally, the original signal is decomposed into several intrinsic mode functions (IMFs) and a residue.

The specific steps of AMD-EEMD method are as follows:

(1) The signal is extracted by AMD method firstly. Assuming there is a time series $x(t)=x_{1}(t)+x_{2}(t)+$ $x_{3}(t)$, the frequencies are $f_{1}, f_{2}, f_{3}$, respectively, and $f_{1}<f_{2}<f_{3}$. If one wants to extract $f_{1}$ frequency component of the signal, take between $f_{1}$ and $f_{2}$ as the bisecting frequency. If one wants to extract $f_{2}$ frequency component of the signal, decompose the first part of the signal which is taking frequency value between $f_{2}$ and $f_{3}$ as the bisecting frequency, and then subtract the first half part of the signal which is taking frequency value between $f_{1}$ and $f_{2}$ as the bisecting frequency.

(2) Assuming that the signal after the extraction is $x_{2}(t)$, one uses EEMD method to decompose it.

(3) Initialize the times of EMD decomposition $M$ and white noise amplitude coefficient $K$, and let $m=1$.

(4) Perform the $m$ time of EMD decomposition. Consider the following:

(a) Add a random sequence of Gauss white noise $n_{i}(t)$ on the input signal $x_{2}(t)$, and then get the signal with noise $x_{2}(t)=x_{2}(t)+k * n_{i}(t)$.

(b) Use EMD to decompose $x_{2}(t)$ and get $I \operatorname{IMF}_{j m}(j=1,2, \ldots, I), j m$ being the decomposition of the first $j$ IMF in the $m$ time of EMD test.

(c) If $m<M$, return to step (4), and let $m=m+1$.

(5) Calculate the mean value of each IMF in $M$ times' test.

(6) Take the output of $\mathrm{IMF}_{j}$ as the first $j \mathrm{IMF}$ decomposed by EEMD.

Usually, $M$ is $100, K$ takes $0.01 \sim 0.5$ times the standard deviation of the original signal which is more appropriate.

2.4. Adaptive Stochastic Resonance and AMD-EEMD Algorithm. Stochastic resonance output SNR formula is defined as follows:

$$
\mathrm{SNR}=10 \lg \frac{S}{N}=10 \lg \frac{2\left|X\left(k_{0}\right)\right|^{2}}{\sum_{k=0}^{L-1}|X(k)|^{2}-2\left|X\left(k_{0}\right)\right|^{2}},
$$

where $S$ is the signal power, $N$ is the noise power, $L$ is data length, and $X\left(k_{0}\right)$ is the unilateral amplitude of output signal frequency component.

When the system input is a small parameter signal, the output SNR approximately is as follows:

$$
\mathrm{SNR}=\frac{\sqrt{2} a^{2} A^{2} \exp \left(-a^{2} / 4 b D\right)}{4 b D^{2}} .
$$

Equation (8) shows that the output SNR is relevant to signal amplitude $A$, noise intensity $D$, and system parameters $a$ and $b$. A signal amplitude as well as the noise intensity is known, so if one wants to get the maximum output SNR one needs to adjust the parameters of $a$ and $b$.

A method of rotating machinery fault diagnosis based on adaptive stochastic resonance and AMD-EEMD is proposed in this paper. Firstly, the bistable stochastic resonance system is optimized by particle swarm optimization (PSO). Secondly, the signal output from the stochastic resonance system is intercepted and decomposed by AMD-EEMD method. The main steps are as follows:

(1) Population initialization: Set up the PSO maximum generation $t_{\max }$, population quantity $M$, learning factors $c_{1}$ and $c_{2}$, upper limit of inertia weight $\omega_{\max }$, lower limit of inertia weight $\omega_{\min }$, upper limit of flight speed $V_{\max }$, lower limit of flight speed $V_{\min }$, and the search range of the system parameters $a$ and $b$. Initialize a group of particles' position and velocity randomly.

(2) The fitness evaluation of each particle: Calculate every particle's fitness value fitness $(x)$ according to the SNR. Store the current position and fitness value of each particle in pbest of each particle. Then, store the position and fitness value of the best individual of all pbest in the gbest.

(3) The velocity and position of particles being updated: The position and velocity of all particles are updated according to (3), and the weight value is updated according to (4). Recalculate every particle's fitness value. If local optimal fitness value pbest or global optimal fitness value gbest of the individual particle is better than the pbest and gbest of the generation particles, then update the corresponding individual particle's local optimal fitness value or global optimal fitness value.

(4) To determine whether the end condition is met: When the evolution times reached the maximum number $t_{\max }$ stop searching. Then output the optimal system parameters $a$ and $b$ and put the original signal into stochastic resonance system with the optimal parameters.

(5) Using the AMD-EEMD method to intercept and decompose the signal which is output from the stochastic resonance system.

The specific process is shown as Figure 1.

\section{Simulation Experiment}

Assuming the input signal is

$$
x(t)=A \sin \left(2 \pi f_{0} t\right)+N(t),
$$

where $A=0.3, f_{0}=0.01 \mathrm{~Hz}$, the strength $D$ of Gauss white noise $N(t)$ is 0.31 , the sampling frequency $f_{s}$ is $5 \mathrm{~Hz}$, the sampling points are 5000, the signal is decomposed by 


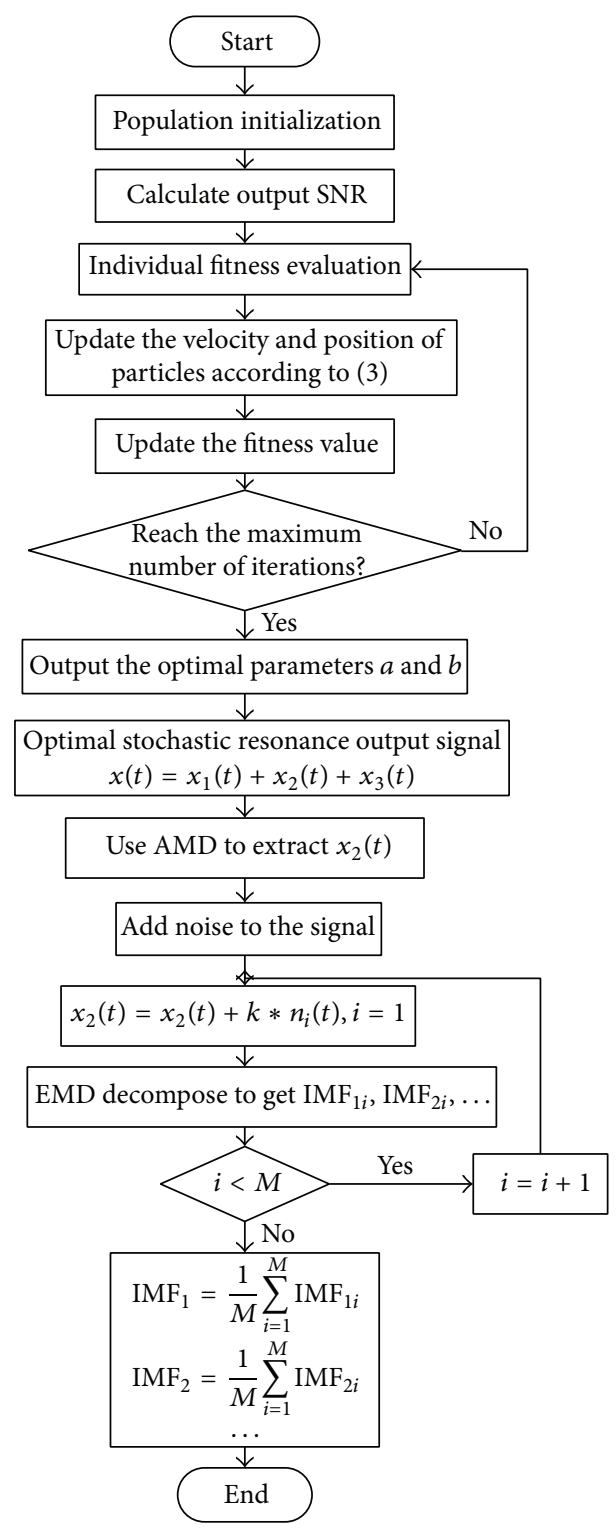

FIGURE 1: The flow chart of adaptive stochastic resonance and AMD-EEMD.

EEMD method directly and the first 8 components are taken, and the result is shown as Figure 2.

First of all, use particle swarm optimization algorithm to optimize parameters $a$ and $b$ of stochastic resonance system. Set the population number as 40, the search range of $a$ and $b$ as $[0,2]$, the maximum value of flight speed as 0.01 , and the maximum number of evolutions as 100. Because the signal amplitude as well as intensity of noise is known, (6) is regarded as the fitness function. From Figure 3 one can get that the maximum output SNR is 0.151 and the optimization result is $a=0.7381$ and $b=0.4393$. Use stochastic resonance system with the optimal parameters to process the original signal, and then get time-domain waveform and frequency spectrum which are shown as Figures 4(c) and 4(d). Figure 4 shows that the noise has been weakened and the $0.01 \mathrm{~Hz}$ frequency component has been greatly improved.

The $0 \sim 0.02 \mathrm{~Hz}$ band part of the original signal and the signal output from stochastic resonance system are extracted by the AMD method, respectively. Then they are decomposed by EEMD method and the frequency spectrum of each component is calculated; the decomposition results are shown as Figures 5 and 6. Comparing with Figure 2, the component is significantly reduced in Figure 5. Figure 5 shows that component $\mathrm{Cl}$ contains the signal whose frequency is $0.01 \mathrm{~Hz}$ and its frequency amplitude is 0.2716 . Figure 6 shows that also component $\mathrm{Cl}$ contains the signal whose frequency is $0.01 \mathrm{~Hz}$, but its frequency amplitude is 1.461. This shows that the original signal is processed by stochastic resonance and then 

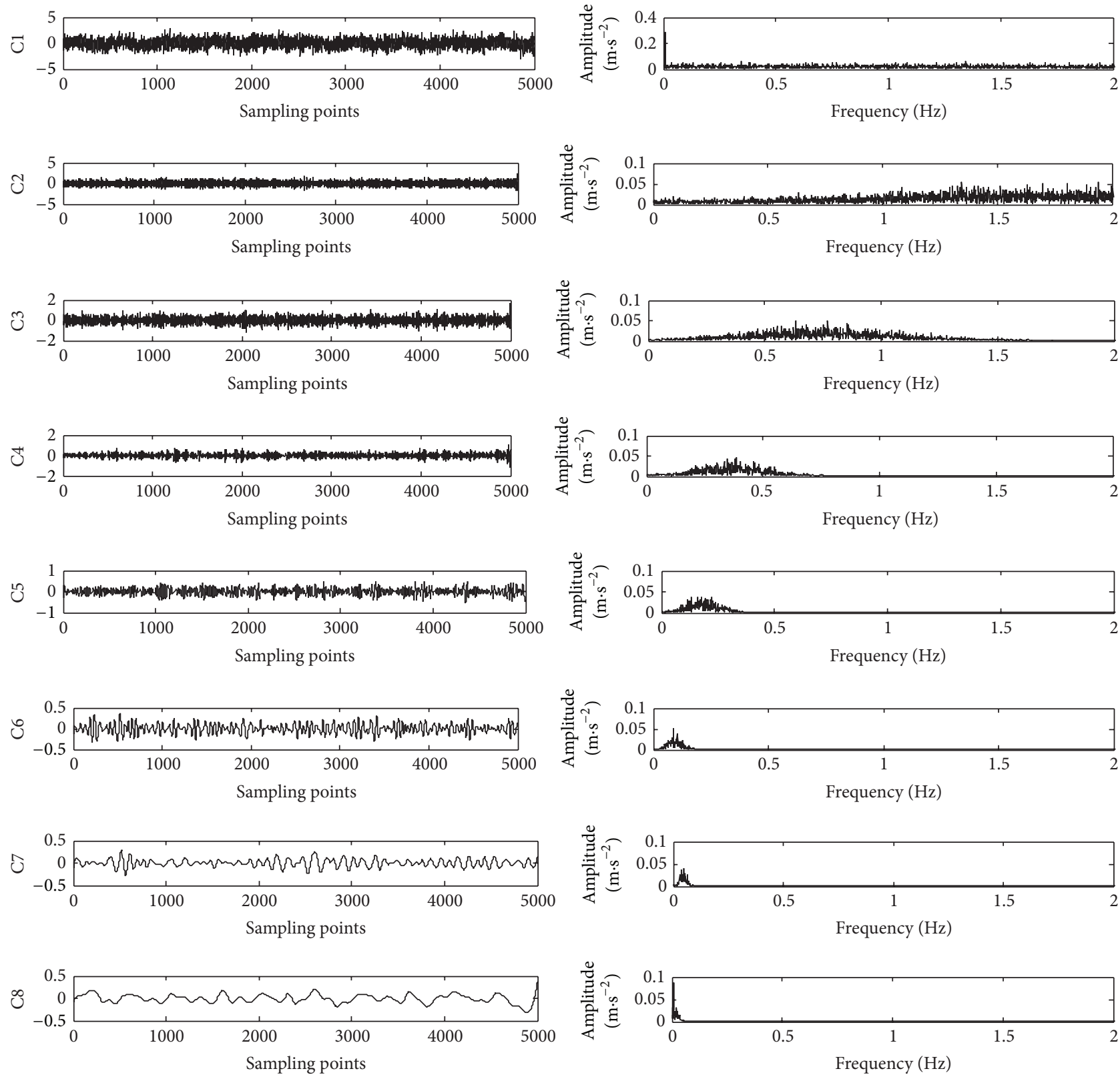

FIGURE 2: The EEMD components of original signal and its spectrum.

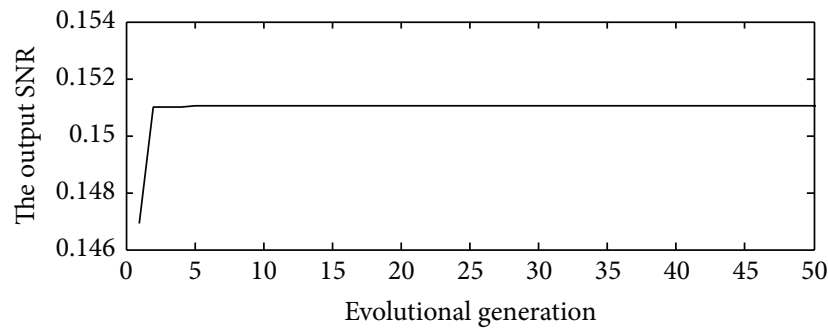

FIGURE 3: The optimal convergence curve of PSO algorithm.

EEMD decomposition, which can make the signal whose frequency is $0.01 \mathrm{~Hz}$ outstanding.

\section{Application Example}

For the Case Western Reserve University rolling bearing fault simulation test bench, the type of drive end bearing is SKF6205, the motor speed is $1797 \mathrm{r} / \mathrm{min}(29.95 \mathrm{~Hz})$, and the sampling frequency is $12 \mathrm{kHz}$. The main parameters of the rolling bearing and the rolling bearing fault feature frequency of the different parts are shown as Tables 1 and 2, respectively.

Take the inner race fault of rolling bearing as an example; select a set of data for analysis and processing. By calculating the feature frequency is $162.18 \mathrm{~Hz}$. The time-domain waveform of the bearing signal and its spectrum are shown as Figure 7. The original signal is decomposed by EEMD method and the first 8 components are taken; the result is shown as Figure 8. 


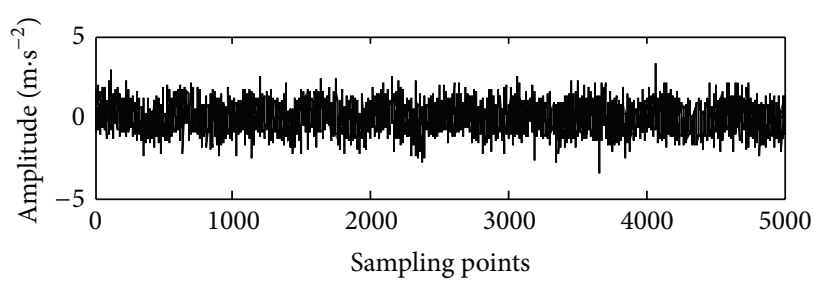

(a) The original signal waveform

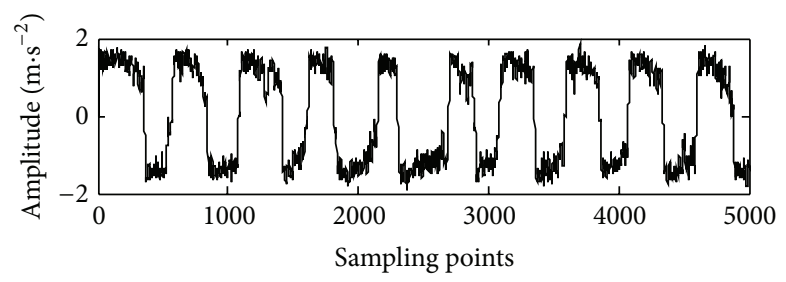

(c) The SR signal waveform

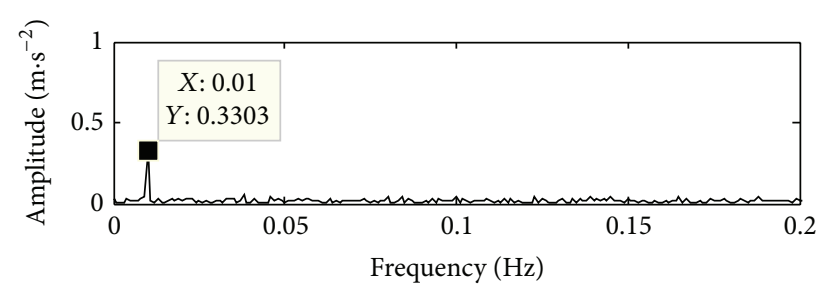

(b) The original signal spectrum

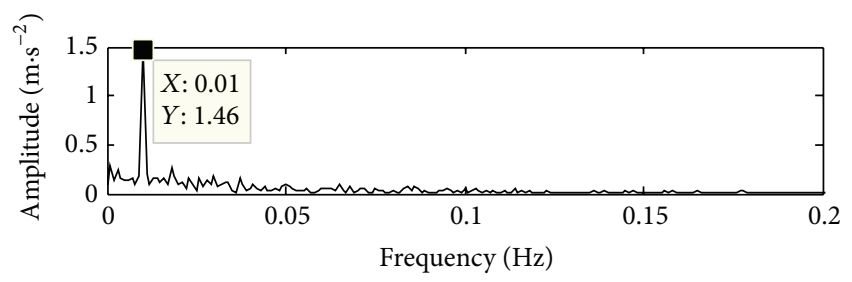

(d) The SR signal spectrum

FIgURE 4: The time-domain waveform and frequency spectrum of original signal and stochastic resonance signal.
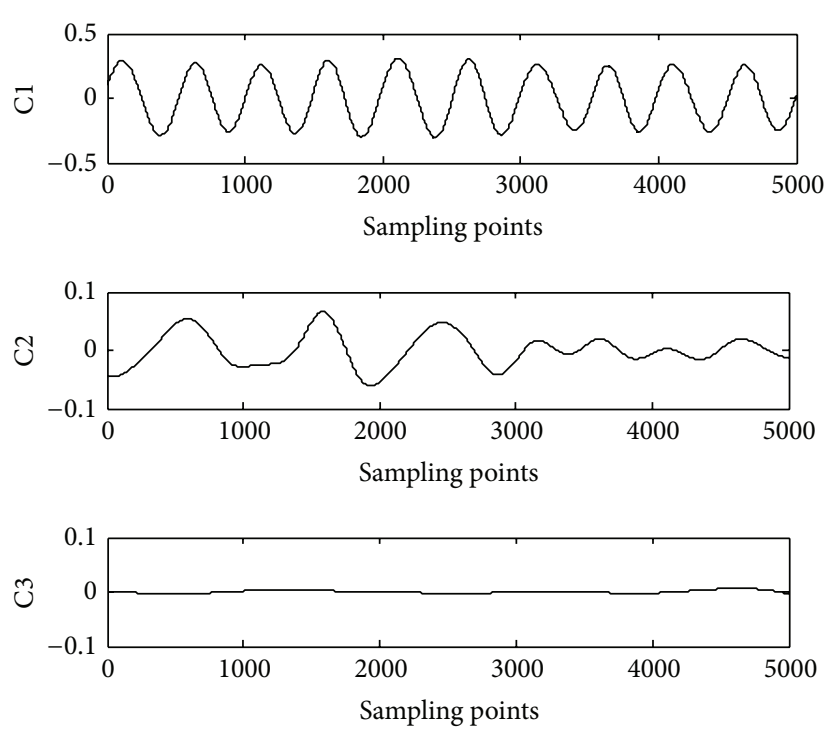
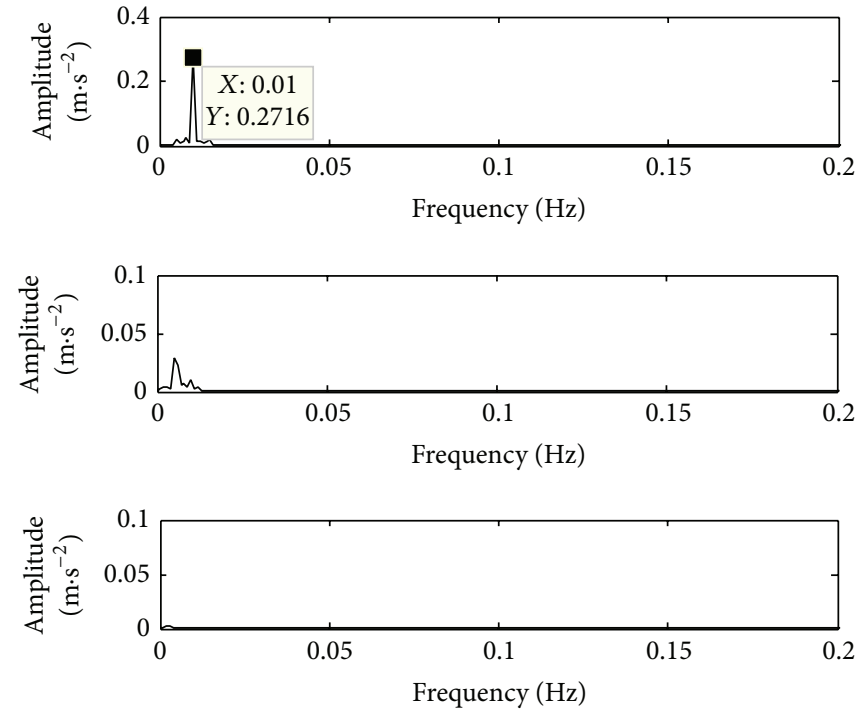

FIgURE 5: The AMD-EEMD components of original signal and its spectrum.

Because the bearing fault signal does not meet the requirements of the small parameter, the signal needs sampling second time. Set up the second sampling frequency $f_{s r}=f_{s} / R=4 \mathrm{~Hz}$; that is to say the transformation scale $R$ is 3000 . Use particle swarm algorithm to optimize the parameters of stochastic resonance system. The noise strength of the bearing fault signal is unknown, so (5) is regarded as the fitness function. After optimizing by PSO algorithm the optimal parameters are $a=0.15$ and $b=2.14$. Then put the bearing signal into stochastic resonance system with the optimal parameters.

The $150 \sim 180 \mathrm{~Hz}$ band part of the original fault signal and the signal output from stochastic resonance system are extracted by the AMD method, respectively. Then they are decomposed by EEMD method and the frequency spectrum of each component is calculated. The decomposition results are shown as Figures 9 and 10. Comparing with Figure 8, the component is significantly reduced in Figure 9.

Figure 9 shows that the main frequency component of $\mathrm{C} 2$ is $162 \mathrm{~Hz}$, according to the inner race fault feature frequency, and its frequency amplitude is 0.01481 . Figure 10 shows that the main frequency component of $\mathrm{C} 2$ is also $162 \mathrm{~Hz}$, but its frequency amplitude is 0.02887 , improving significantly compared with Figure 9.

The comparative analysis outlined above indicates that the adaptive stochastic resonance and AMD-EEMD method may not only increase the output SNR but also reduce the effective components of EEMD and improve the amplitude of components frequency. Therefore, the adaptive stochastic resonance and AMD-EEMD method and its application in fault diagnosis of rotating machinery have practical significance. 

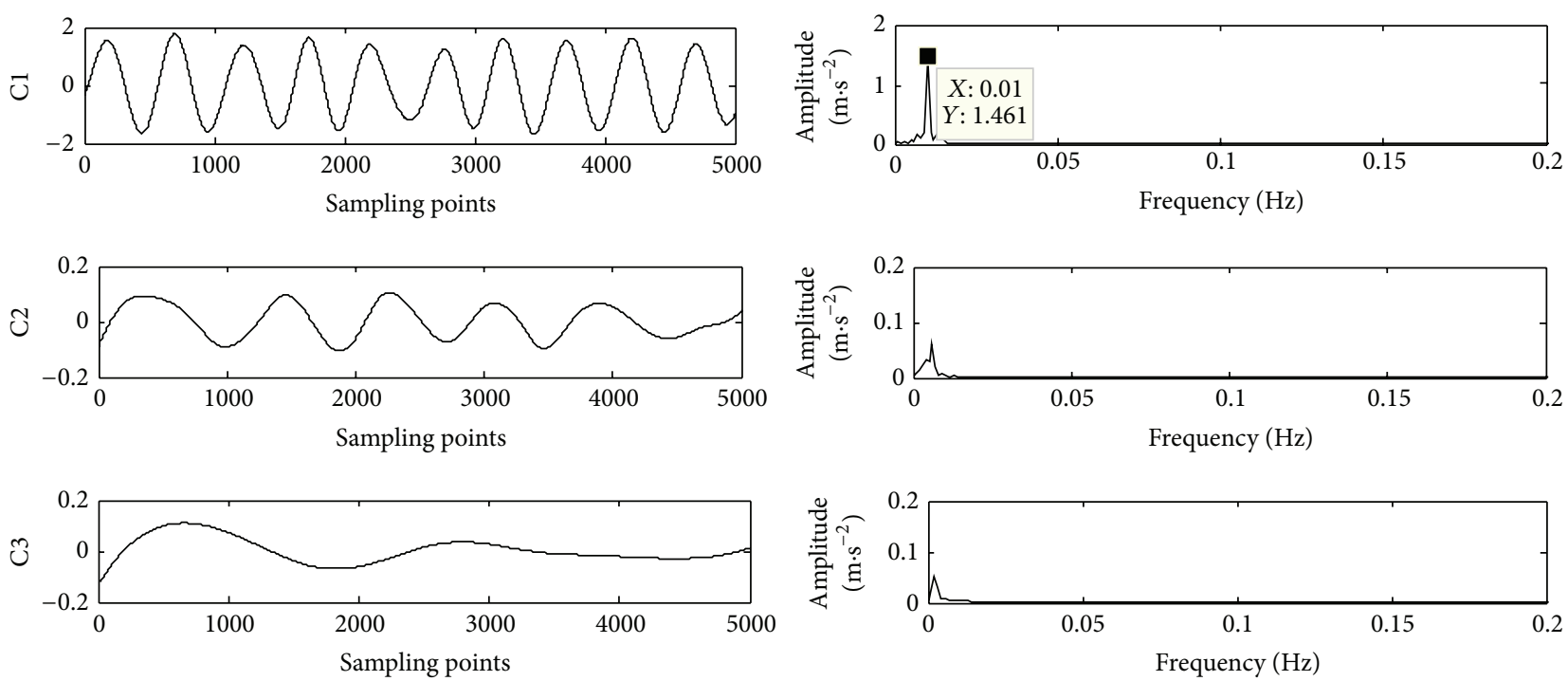

FIGURE 6: The AMD-EEMD components of stochastic resonance signal and its spectrum.

TABLE 1: The main parameters of the rolling bearing of SKF6205.

\begin{tabular}{lccccc}
\hline Out diameter $R(\mathrm{~mm})$ & Inner diameter $r(\mathrm{~mm})$ & Pitch diameter $D(\mathrm{~mm})$ & Ball diameter $d(\mathrm{~mm})$ & Ball number $Z$ & Contact angle $a$ \\
\hline 52.00 & 25.00 & 39.04 & 7.94 & 9 & $0^{\circ}$ \\
\hline
\end{tabular}

TABLE 2: Rolling bearing fault feature frequency of SKF6205.

\begin{tabular}{lcccc}
\hline Bearing element & Inner ring & Outer ring & The retainer & Rolling body \\
\hline Feature frequency & $5.4152 f_{r}$ & $3.5848 f_{r}$ & $0.39828 f_{r}$ & $4.7135 f_{r}$ \\
\hline
\end{tabular}

Note. $f_{r}=N / 60$ and $N$ is bearing speed.

For the Case Western Reserve University rolling bearing fault simulation test bench, the other type of fan end bearing is SKF6203 and the sampling frequency is $12 \mathrm{kHz}$. The main parameters of the rolling bearing and the rolling bearing fault feature frequency of the different parts are shown as Tables 3 and 4 , respectively.

Forty-four groups of bearing inner fault data were analyzed by EMD, EEMD, SR-EMD, SR-EEMD, AMD-EMD, AMD-EEMD, and SR-AMD-EEMD method, respectively. Among them, forty-two groups of data can effectively increase the amplitude and reduce the number of components after SR-AMD-EEMD method; that is, the accuracy of this method is $95.45 \%$.

Here take eight groups of bearing inner race fault of SKF6205 and SKF6203 that are analyzed under $1797 \mathrm{r} / \mathrm{min}$, $1772 \mathrm{r} / \mathrm{min}, 1750 \mathrm{r} / \mathrm{min}$, and $1730 \mathrm{r} / \mathrm{min}$ different speed, respectively. The frequency amplitude and component count are shown in Tables 5 and 6. From Tables 5 and 6, we can clearly see that the EMD and EEMD methods have similar amplitude and number of components, the SR-EMD and SREEMD methods increase the amplitude, but the number of the components is not changed, the AMD-EMD and AMDEEMD methods reduce the number of components, but the amplitude is almost unchanged, and the SR-AMD-EEMD method has the largest amplitude, while the component is the least. This shows the effectiveness of the proposed method in this paper.

Calculate the average value of the amplitude increment of eight groups which is compared with the EMD method. The percentage increase is shown in Figure 11. And the average number of components is shown in Figure 12. Figure 11 shows that the amplitude increased by about $10 \%$ after EEMD, AMD-EMD, and AMD-EEMD method compared with EMD method. And the amplitude increased by $96.74 \%, 116.10 \%$, and $122.58 \%$ after SR-EMD, SR-EEMD, and SR-AMD-EEMD method, respectively. From Figure 12 we can see that the minimum average number of components is 3 processed by the SR-AMD-EEMD method. To sum up we know that the SR-AMD-EEMD method has the largest amplitude and the minimum number of components.

\section{Conclusions}

Consider the following:

(1) This paper presents an adaptive stochastic resonance and AMD-EEMD method for fault diagnosis of rotating machinery. The process is as follows: firstly, the bistable stochastic resonance system is optimized by PSO, and the best structure parameters are obtained; 

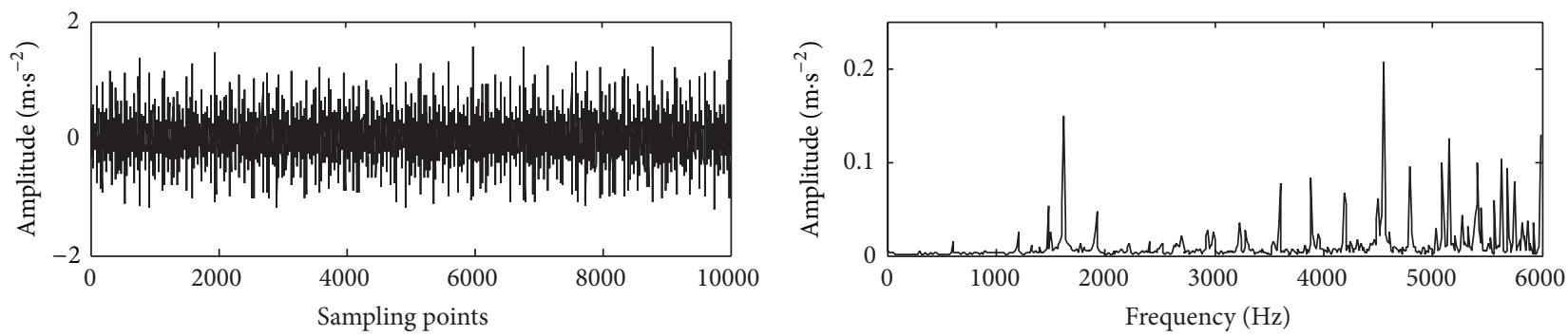

FIGURE 7: The bearing fault signal and its spectrum.
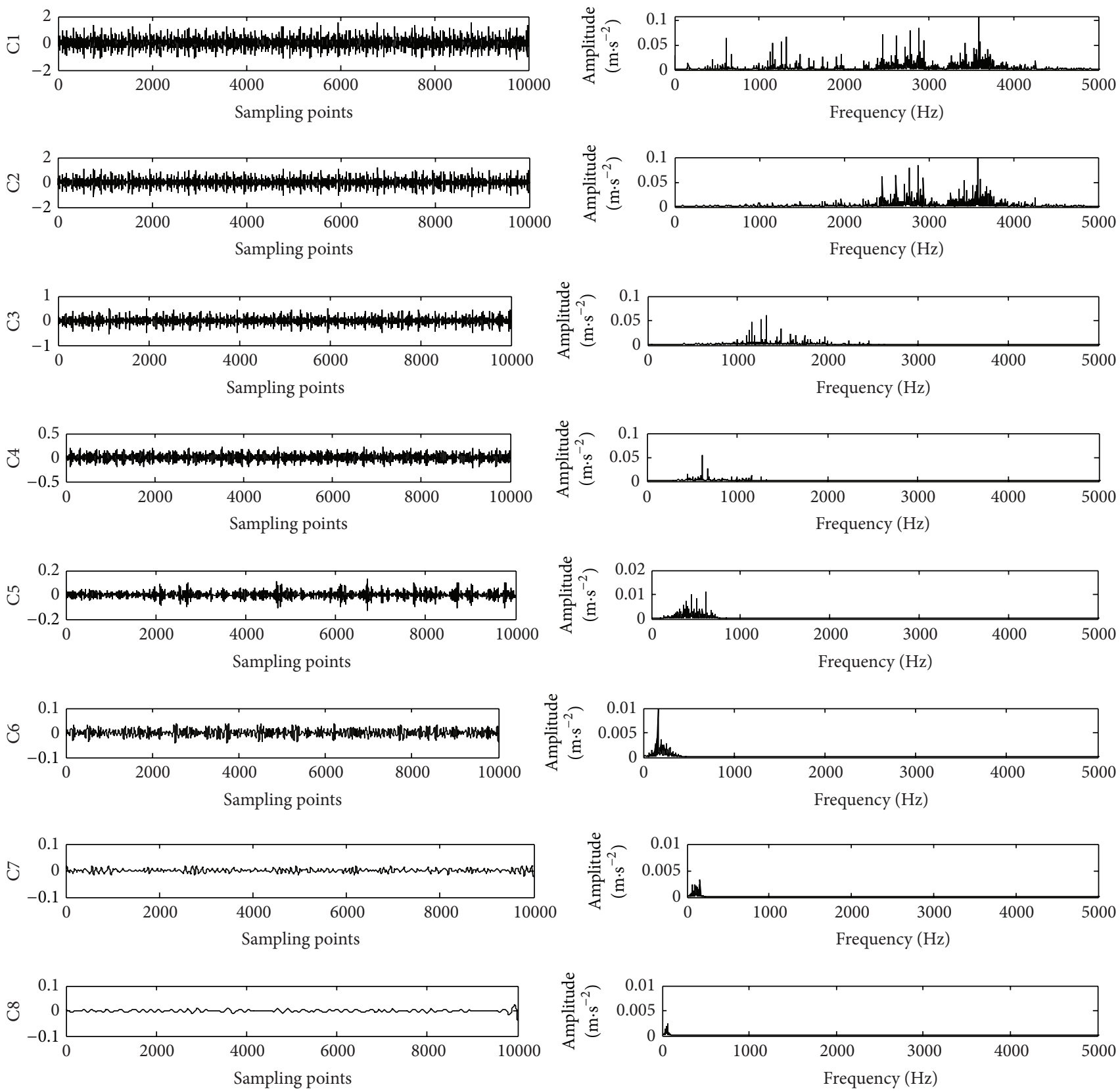

FIGURE 8: The EEMD components of original fault signal and its spectrum. 

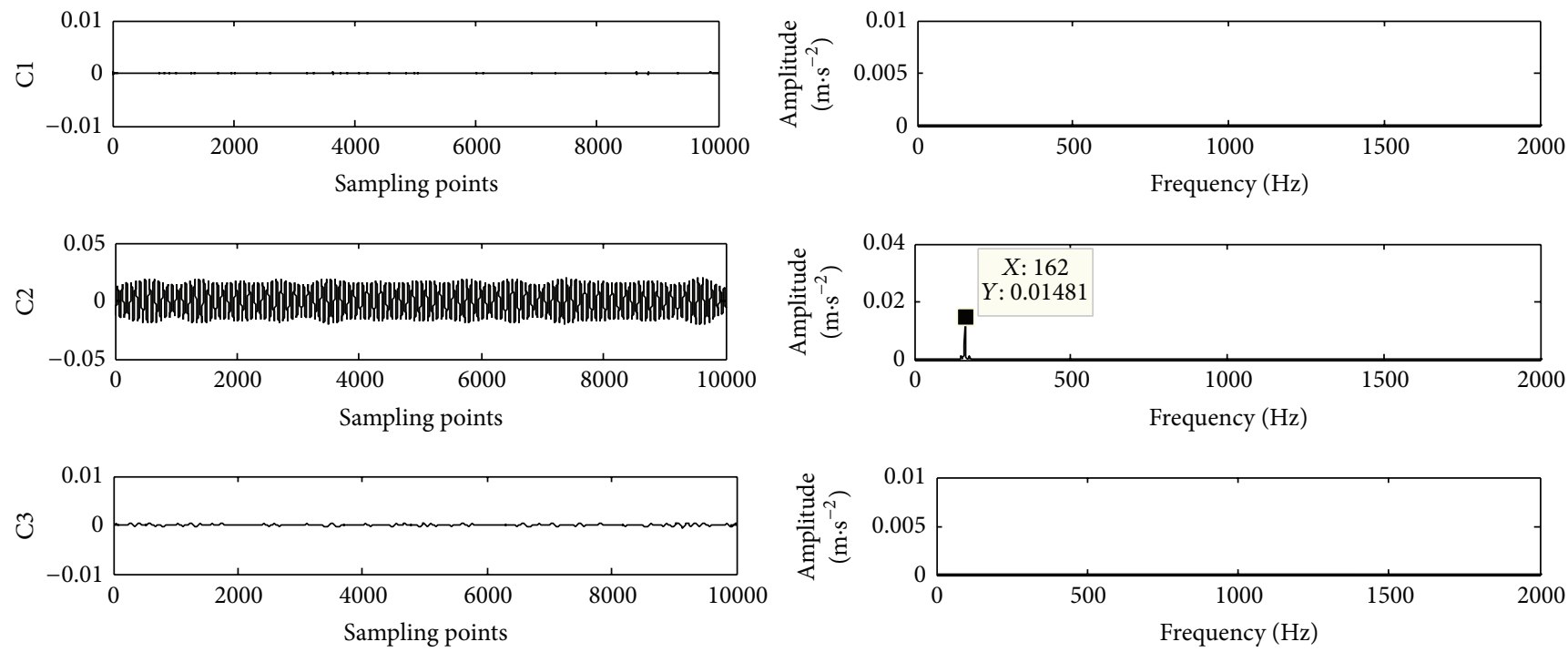

FIGURE 9: The AMD-EEMD components of original fault signal and its spectrum.
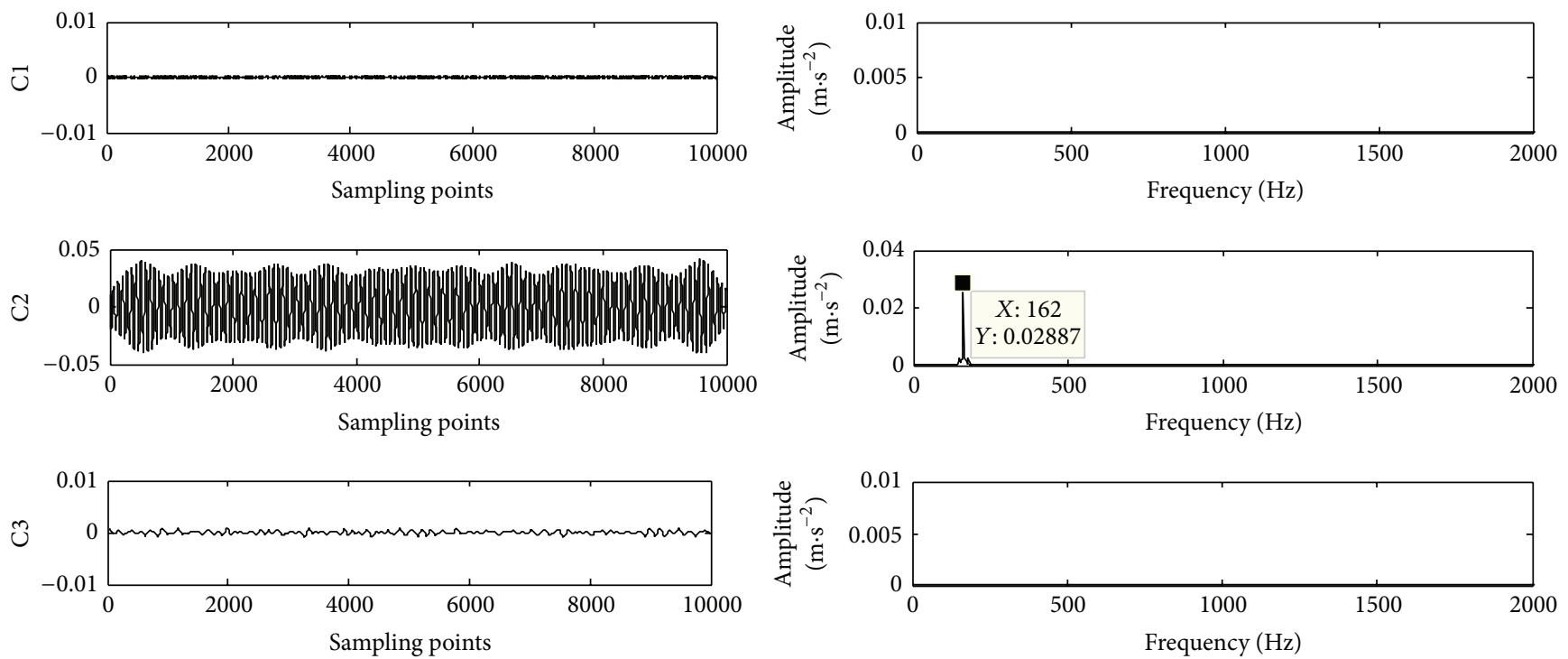

FIGURE 10: The AMD-EEMD components of stochastic resonance signal and its spectrum.

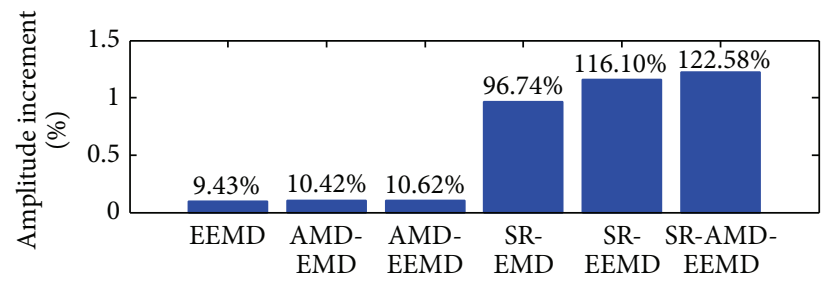

FIGURE 11: The column chart of percentage increase.

then, the signal with noise is put into the stochastic resonance system in order to improve the SNR of the signal; secondly, the signal output from the stochastic resonance system is extracted by AMD method; finally, the signal is decomposed by EEMD method.

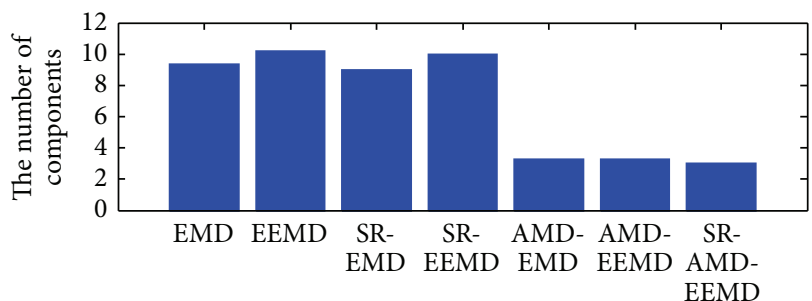

FIGURE 12: The average number of components.

(2) By processing the simulation signal and application examples and comparing the SR-AMD-EEMD method and EMD, EEMD, SR-EMD, SR-EEMD, AMD-EMD, and AMD-EEMD method, respectively, 
TABLE 3: The main parameters of the rolling bearing of SKF6203.

\begin{tabular}{lccccc}
\hline Out diameter $R(\mathrm{~mm})$ & Inner diameter $r(\mathrm{~mm})$ & Pitch diameter $D(\mathrm{~mm})$ & Ball diameter $d(\mathrm{~mm})$ & Ball number $Z$ & Contact angle $a$ \\
\hline 40.00 & 17.00 & 28.50 & 6.75 & 8 & $0^{\circ}$ \\
\hline
\end{tabular}

TABLE 4: Rolling bearing fault feature frequency of SKF6203.

\begin{tabular}{lcccc}
\hline Bearing element & Inner ring & Outer ring & The retainer & Rolling body \\
\hline Feature frequency & $4.9469 f_{r}$ & $3.0530 f_{r}$ & $0.3817 f_{r}$ & $3.9874 f_{r}$ \\
\hline
\end{tabular}

Note. $f_{r}=N / 60$ and $N$ is bearing speed.

TABLE 5: The amplitude and component count of fault feature frequency of SKF6205 under different rotational speed.

\begin{tabular}{|c|c|c|c|c|c|c|c|c|}
\hline \multirow{2}{*}{$\begin{array}{l}\text { Bearing type } \\
\text { Motor speed }\end{array}$} & \multicolumn{8}{|c|}{ SKF6205 } \\
\hline & \multicolumn{2}{|c|}{$1797 \mathrm{r} / \mathrm{min}$} & \multicolumn{2}{|c|}{$1772 \mathrm{r} / \mathrm{min}$} & \multicolumn{2}{|c|}{$1750 \mathrm{r} / \mathrm{min}$} & \multicolumn{2}{|c|}{$1730 \mathrm{r} / \mathrm{min}$} \\
\hline \multirow[t]{2}{*}{ Frequency value } & \multicolumn{2}{|c|}{$162 \mathrm{~Hz}$} & \multicolumn{2}{|c|}{$159.6 \mathrm{~Hz}$} & \multicolumn{2}{|c|}{$157.2 \mathrm{~Hz}$} & \multicolumn{2}{|c|}{$154.8 \mathrm{~Hz}$} \\
\hline & Amplitude & $\begin{array}{c}\text { Component } \\
\text { count }\end{array}$ & Amplitude & $\begin{array}{c}\text { Component } \\
\text { count }\end{array}$ & Amplitude & $\begin{array}{c}\text { Component } \\
\text { count }\end{array}$ & Amplitude & $\begin{array}{c}\text { Component } \\
\text { count }\end{array}$ \\
\hline SR-AMD-EEMD & 0.02887 & 3 & 0.02164 & 2 & 0.02567 & 3 & 0.02608 & 2 \\
\hline AMD-EEMD & 0.01481 & 3 & 0.01402 & 2 & 0.01518 & 3 & 0.00881 & 3 \\
\hline AMD-EMD & 0.01536 & 3 & 0.01336 & 2 & 0.01528 & 4 & 0.00863 & 2 \\
\hline SR-EEMD & 0.02880 & 11 & 0.02034 & 10 & 0.02555 & 10 & 0.02480 & 9 \\
\hline SR-EMD & 0.02579 & 9 & 0.01738 & 9 & 0.02001 & 8 & 0.02729 & 10 \\
\hline EEMD & 0.01324 & 10 & 0.01672 & 10 & 0.01314 & 10 & 0.00834 & 11 \\
\hline EMD & 0.01163 & 10 & 0.01371 & 9 & 0.01224 & 9 & 0.00763 & 10 \\
\hline
\end{tabular}

TABLE 6: The amplitude and component count of fault feature frequency of SKF6203 under different rotational speed.

\begin{tabular}{|c|c|c|c|c|c|c|c|c|}
\hline \multirow{2}{*}{$\begin{array}{l}\text { Bearing type } \\
\text { Motor speed }\end{array}$} & \multicolumn{8}{|c|}{ SKF6203 } \\
\hline & \multicolumn{2}{|c|}{$1797 \mathrm{r} / \mathrm{min}$} & \multicolumn{2}{|c|}{$1772 \mathrm{r} / \mathrm{min}$} & \multicolumn{2}{|c|}{$1750 \mathrm{r} / \mathrm{min}$} & \multicolumn{2}{|c|}{$1730 \mathrm{r} / \mathrm{min}$} \\
\hline \multirow[t]{2}{*}{ Frequency value } & \multicolumn{2}{|c|}{$147.6 \mathrm{~Hz}$} & \multicolumn{2}{|c|}{$146.4 \mathrm{~Hz}$} & \multicolumn{2}{|c|}{$144 \mathrm{~Hz}$} & \multicolumn{2}{|c|}{$142.8 \mathrm{~Hz}$} \\
\hline & Amplitude & $\begin{array}{c}\text { Component } \\
\text { count }\end{array}$ & Amplitude & $\begin{array}{c}\text { Component } \\
\text { count }\end{array}$ & Amplitude & $\begin{array}{c}\text { Component } \\
\text { count }\end{array}$ & Amplitude & $\begin{array}{c}\text { Component } \\
\text { count }\end{array}$ \\
\hline SR-AMD-EEMD & 0.01287 & 4 & 0.00858 & 3 & 0.00754 & 3 & 0.00748 & 4 \\
\hline AMD-EEMD & 0.00524 & 4 & 0.00342 & 3 & 0.00323 & 4 & 0.00375 & 4 \\
\hline AMD-EMD & 0.00529 & 4 & 0.00345 & 3 & 0.00335 & 4 & 0.00382 & 4 \\
\hline SR-EEMD & 0.01208 & 10 & 0.00854 & 10 & - & - & - & - \\
\hline SR-EMD & 0.01228 & 9 & 0.00660 & 9 & - & - & - & - \\
\hline EEMD & 0.00574 & 10 & 0.00364 & 10 & - & - & - & - \\
\hline EMD & 0.00568 & 9 & 0.00469 & 9 & - & - & - & - \\
\hline
\end{tabular}

Note. “-” expresses no result.

the result shows that the SR-AMD-EEMD method can effectively improve the SNR and also reduce the number of EEMD effective components, thus improving the quality and accuracy of EEMD decomposition and enhancing the amplitude of components frequency. Therefore, it is valuable in engineering applications.

\section{Conflict of Interests}

The authors declare that there is no conflict of interests regarding the publication of this paper.

\section{Acknowledgments}

This work was supported by the National Natural Science Foundation of China (Grant no. 51475407), Hebei Provincial Natural Science Foundation of China (no. E2015203190), and Key Project of Natural Science Research in Colleges and Universities of Hebei Province (Grant no. ZD2015050).

\section{References}

[1] Y. Tian, J. Ma, C. Lu, and Z. Wang, "Rolling bearing fault diagnosis under variable conditions using LMD-SVD and 
extreme learning machine," Mechanism and Machine Theory, vol. 90, pp. 175-186, 2015.

[2] J. Zheng, J. Cheng, and Y. Yang, "A rolling bearing fault diagnosis approach based on LCD and fuzzy entropy," Mechanism \& Machine Theory, vol. 70, no. 6, pp. 441-453, 2013.

[3] H. Liu and M. Han, "A fault diagnosis method based on local mean decomposition and multi-scale entropy for roller bearings," Mechanism \& Machine Theory, vol. 75, pp. 67-78, 2014.

[4] J. Zheng, J. Cheng, Y. Yang, and S. Luo, "A rolling bearing fault diagnosis method based on multi-scale fuzzy entropy and variable predictive model-based class discrimination," Mechanism and Machine Theory, vol. 78, pp. 187-200, 2014.

[5] R. Benzi, A. Sutera, and A. Vulpiani, "The mechanism of stochastic resonance," Journal of Physics A: Mathematical and General, vol. 14, no. 11, pp. L453-L457, 1981.

[6] Y. Hang and H. Yan, "Research on weak signal detection based on cascaded stochastic bistable resonance," Computer Measurement \& Control, vol. 20, no. 7, pp. 1821-1836, 2012.

[7] P. Shi, X. Ding, and D. Han, "Study on multi-frequency weak signal detection method based on stochastic resonance tuning by multi-scale noise," Measurement, vol. 47, no. 1, pp. 540-546, 2014.

[8] Y. G. Leng and T. Y. Wang, "Numerical research of twice sampling stochastic resonance for the detection of a weak signal submerged in a heavy noise," Acta Physica Sinica, vol. 52, no. 10, pp. 2432-2437, 2003.

[9] S. Lu, Q. He, and F. Kong, "Effects of underdamped step-varying second-order stochastic resonance for weak signal detection," Digital Signal Processing, vol. 36, pp. 93-103, 2015.

[10] Q. He and J. Wang, "Effects of multiscale noise tuning on stochastic resonance for weak signal detection," Digital Signal Processing, vol. 22, no. 4, pp. 614-621, 2012.

[11] L. Zhang, F. Wang, and Z. Hui, "Detecting high frequency weak signal using parameter-adjusted frequency shifting based on stochastic resonance (SR)," Physics Procedia, vol. 24, pp. 382389, 2012.

[12] N. E. Huang, Z. Shen, S. R. Long et al., "The empirical mode decomposition and the Hilbert spectrum for nonlinear and non-stationary time series analysis," Proceedings of the Royal Society of London, Series A: Mathematical, Physical and Engineering Sciences, vol. 454, no. 1971, pp. 903-995, 1998.

[13] Z. Wu and N. E. Huang, "Ensemble empirical mode decomposition: a noise-assisted data analysis method," Advances in Adaptive Data Analysis, vol. 1, no. 1, pp. 1-49, 2011.

[14] Y.-H. Wang, C.-H. Yeh, H.-W. V. Young, K. Hu, and M.-T. Lo, "On the computational complexity of the empirical mode decomposition algorithm," Physica A: Statistical Mechanics \& Its Applications, vol. 400, no. 2, pp. 159-167, 2014.

[15] W. Jin-Dun, C. Lue, and Q. Wei, Research on Fault Weak Signal Feature Extraction Based on EEMD Method, Electronic Design Engineering, Norco, Calif, USA, 2012.

[16] Y. Lei, N. Li, J. Lin, and S. Wang, "Fault diagnosis of rotating machinery based on an adaptive ensemble empirical mode decomposition," Sensors, vol. 13, no. 12, pp. 16950-16964, 2013.

[17] F. Zhao, X. Mei, T. Tao, G. Jiang, and Y. Zhou, "Fault diagnosis of a machine tool rotary axis based on a motor current test and the ensemble empirical mode decomposition method," Proceedings of the Institution of Mechanical Engineers, Part C: Journal of Mechanical Engineering Science, vol. 225, no. 5, pp. 1121-1129, 2011.
[18] G. Chen and Z. Wang, "A signal decomposition theorem with Hilbert transform and its application to narrowband time series with closely spaced frequency components," Mechanical Systems and Signal Processing, vol. 28, pp. 258-279, 2012.

[19] S. Fauve and F. Heslot, "Stochastic resonance in a bistable system," Physics Letters A, vol. 97, no. 1-2, pp. 5-7, 1983.

[20] Y.-G. Leng, T.-Y. Wang, Y. Guo, Y.-G. Xu, and S.-B. Fan, "Engineering signal processing based on bistable stochastic resonance," Mechanical Systems \& Signal Processing, vol. 21, no. 1, pp. 138-150, 2007.

[21] Y. Qin, Y. Tao, Y. He, and B. Tang, "Adaptive bistable stochastic resonance and its application in mechanical fault feature extraction," Journal of Sound and Vibration, vol. 333, no. 26, pp. 73867400, 2014.

[22] J. Kennedy and R. Eberhart, "Particle swarm optimization," in Proceedings of the IEEE International Conference on Neural Networks, vol. 4, pp. 129-132, Perth, Australia, December 1995.

[23] Y. Shi and R. C. Eberhart, "Parameter selection in particle swarm optimization," in Evolutionary Programming VII, vol. 1447 of Lecture Notes in Computer Science, pp. 591-600, Springer, Berlin, Germany, 1998. 


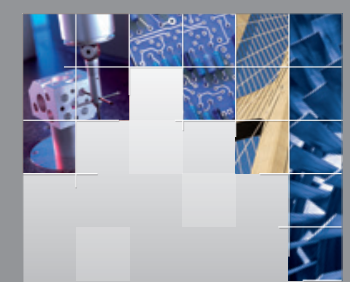

\section{Enfincering}
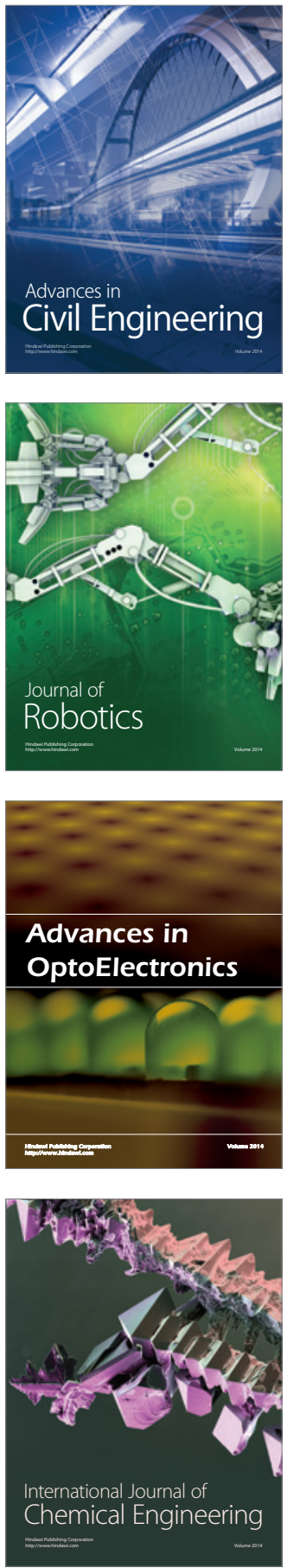

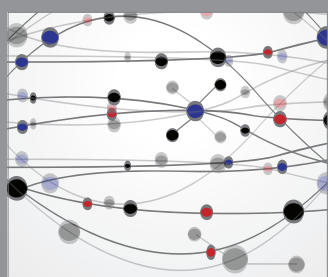

The Scientific World Journal

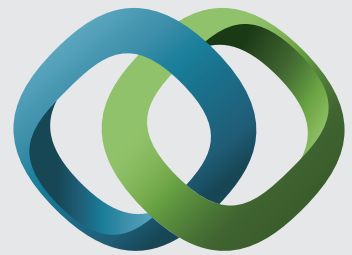

\section{Hindawi}

Submit your manuscripts at

http://www.hindawi.com
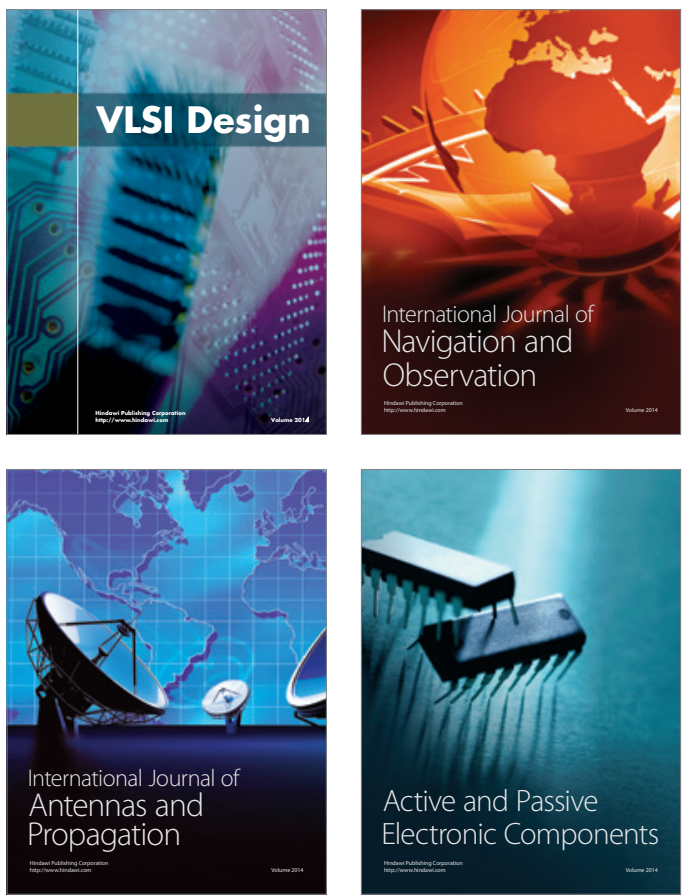
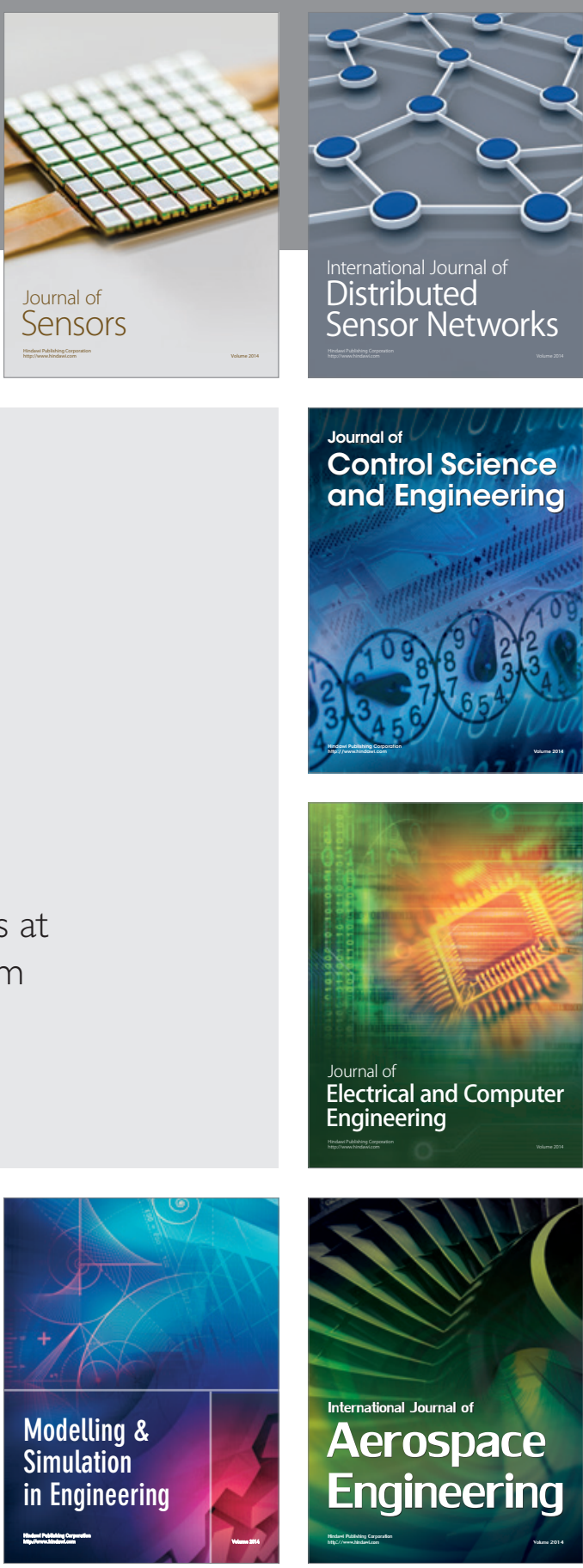

International Journal of

Distributed

Sensor Networks

Journal of

Control Science

and Engineering
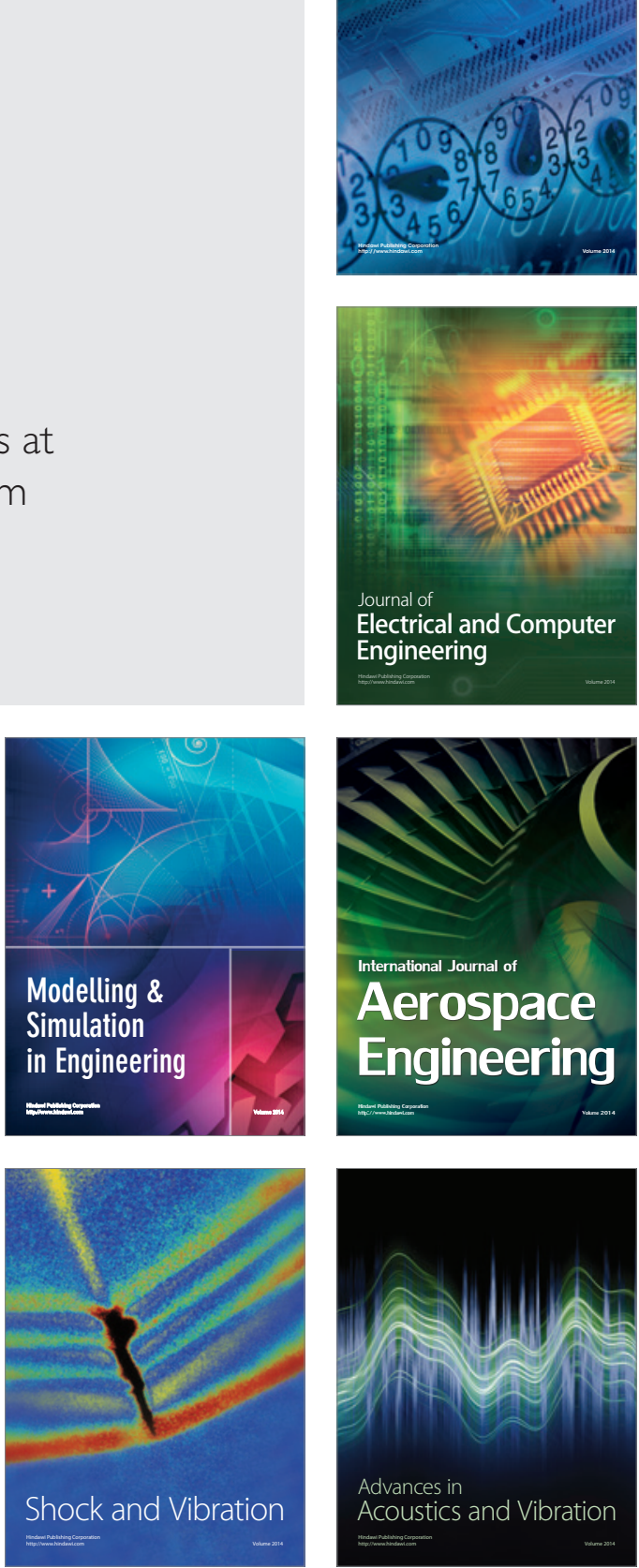